Pacific Journal of Mathematics

MAPPINGS BETWEEN ANRS THAT ARE FINE HOMOTOPY

WIUtam Ever Haver 


\title{
MAPPINGS BETWEEN ANRs THAT ARE FINE HOMOTOPY EQUIVALENCES
}

\author{
William E. Haver
}

\begin{abstract}
It is shown in this note that every closed $U V^{\infty}-\operatorname{map}$ between separable ANRs is a fine homotopy equivalence.
\end{abstract}

We extend Lacher's result $[6,7]$ that a closed $U V^{\infty}$-map between locally compact, finite dimensional ANRs is a fine homotopy equivalence to the case of arbitrary separable ANRs. It is hoped that this theorem will be useful in studying manifolds modelled on the Hilbert Cube. (See [1], section PF3. Added in proof. See also [9]).

A set $A \subset X$ has property $U V^{\infty}$ if for each open set $U$ of $X$ containing $A$, there is an open $V$, with $A \subset V \subset U$ such that $V$ is null-homotopic in $U$. A mapping $f: X \rightarrow Y$ of $X$ onto $Y$ is a $U V^{\infty}$-map if for each $y \in Y, f^{-1}(y)$ is a $U V^{\infty}$ subset of $X$. The mapping $f$ is said to be closed if the image of every closed set is closed and proper if the inverse image of every compact set is compact. An absolute neighborhood retract for metric spaces is denoted an ANR. If $\alpha$ is a cover of $Y$ and $g_{1}$ and $g_{2}$ are maps of a space $A$ into $Y, g_{1}$ is $\alpha$-near $g_{2}$ if for each $a \in A$ there is a $U \in \alpha$ containing $g_{1}(a)$ and $g_{2}(a)$. The map $g_{1}$ is $\alpha$-homotopic to $g_{2}, g_{1} \stackrel{\alpha}{\simeq} g_{2}$, if there is a homotopy $\lambda: A \times I \rightarrow Y$ taking $g_{1}$ to $g_{2}$ with the property that for each $a \in A$ there exists $U \in \alpha$ containing $\lambda(\{a\} \times I)$. A map $f: X \rightarrow Y$ is a fine homotopy equivalence if for each open cover, $\alpha$, of $Y$ there exists a map $g: Y \rightarrow X$ such that $f g \stackrel{\alpha}{\simeq} i d_{Y}$ and $g f \stackrel{f^{\prime \prime}(\alpha)}{=} i d_{X}$.

Various versions of Lemma 3 have been proven by Smale [8], Armentrout and Price [2], Kozlowski [5] and Lacher [6]. The difference in this lemma is that $K$ is not required to be a finite dimensional complex.

Let $K$ be a locally finite complex and $j$ be a nonnegative integer. When there is no confusion we will not distinghish between the complex $K$ and its underlying point set $|K|$. If $\sigma$ is a simplex of $K$, then $\quad N(\sigma, K)=\{\tau<K \mid \sigma \cap \tau \neq \phi\} \quad$ and $\operatorname{st}(\sigma, K)=$ $\{\tau<K \mid \sigma<\tau\}$. Also $K^{i}$ will denote the $j$-skeleton of $K$ and ${ }^{i} K=$ $\left\{\sigma<K|| N(\sigma, K)|\subset| K^{j} \mid\right\}$. Let $\mathcal{U}$ be a covering of a space $Y$ and $B$ a subset of $Y$. The star of $B$ with respect to $U$, $\operatorname{st}^{1}(B, \mathcal{U})$, is the set $\{U \in U \mid B \cap U \neq \phi\}$. Inductively, $\operatorname{st}^{n}(B, \mathcal{U})$ is defined to be $\operatorname{st}\left(\operatorname{st}^{n-1}(B, \mathcal{U})\right)$. A covering $\mathscr{V}$ is called a $\operatorname{star}^{n}$ refinement of $\mathscr{U}$ if the covering $\left\{\mathrm{st}^{n}(V, \mathscr{V}) / V \in V\right\}$ refines $\mathcal{U}$. Every open covering of a 
metric space has an open $\operatorname{star}^{n}$ refinement for each positive integer $n$ (c.f. [3]). We start by stating without proof two easily verified lemmas.

Lemma 1. Let $K$ be a locally finite complex. Suppose $\phi: K \rightarrow Y$ is a map, $U$ is an open cover of $Y$, and $k$ is a nonnegative integer. Then there is a subdivision $\tilde{K}$ of $K$ so that:

(a) if $\sigma$ is a k-simplex of $\tilde{K}$, then $\phi(N(\sigma, \tilde{K})) \subset U$, for some $U \in U$,

(b) if $\sigma<^{k-1} K$, then $\sigma<\tilde{K}$.

We will call such a subdivision, $\tilde{K}, a(k, \mathcal{U})$-subdivision of $K$. We note that for any vertex, $v$, of $\tilde{K}$ with $v \notin^{k-1} K$ it follows that $\phi(\operatorname{st}(v, \tilde{K})) \subset U$ for some $U \in \mathcal{U}$.

LEMMA 2. Let $U$ be an open cover of the paracompact space $Y$ and $f: X \rightarrow Y$ a closed $U V^{\infty}$-map. Then there is an open locally finite refinement $\mathscr{V}$ of $\mathcal{U}$ such that for each $V \in \mathcal{V}$, there is a $U \in \mathcal{U}$ satisfying

(a) $\operatorname{st}(V, \mathscr{V}) \subset U$

(b) if $m$ is a positive integer and the map $\gamma: \partial B^{m} \rightarrow f^{-1}(\operatorname{st}(V, \mathscr{V}))$ is given, then $\gamma$ can be extended to $\bar{\gamma}: B^{m} \rightarrow f^{-1}(U)$.

We will call such a refinement, $\mathcal{V}$, a $U V^{\infty}$ star refinement of $\mathcal{U}$.

Lemma 3. Let $f: X \rightarrow Y$ be a closed $U V^{\infty}$-map of an arbitrary space, $X$, onto the paracompact space $Y$. Let $K$ be a locally finite complex and $J$ a subcomplex of $K$. Let $\phi: K \rightarrow Y$ and $\psi^{\prime}: J \rightarrow X$ be mappings such that $f \psi^{\prime}=\phi \mid J$. Then given any open cover, $\alpha$, of $Y$ there exists a map $\psi: K \rightarrow X$ extending $\psi^{\prime}$ so that $f \psi$ is $\alpha$-near $\phi$.

Proof. Let $K_{0}$ be a $(0, \alpha)$-subdivision of $K$ and let $\alpha_{0}=\alpha$. Define inductively a sequence of covers of $Y,\left\{\alpha_{i}\right\}_{i=0}^{\infty}$, and subdivisions of $K_{0}$, $\left\{K_{i}\right\}_{i=0}^{\infty}$, such that for each $i>0, \alpha_{i}$ is a $U V^{\infty}$ star refinement of $\alpha_{i-1}$ and $K_{i}$ is an $\left(i, \alpha_{i}\right)$-subdivision of $K_{i-1}$.

Define $\psi_{0}: K_{0}^{0} \rightarrow X$ by letting $\psi_{0}(v)=\psi^{\prime}(v)$ if $V \in J$ and otherwise an arbitrary element of $f^{-1}(\phi(v))$. Assume inductively that there exist maps $\left\{\psi_{i}: K_{i}^{i} \rightarrow X\right\}_{i=0}^{n}$ such that for $0 \leqq i \leqq n$ :

(1) $\psi_{i}\left|J \cap K_{i}^{i}=\psi^{\prime}\right| J \cap K_{i}^{i}$ and if $j<i,\left.\psi_{i}\right|^{i} K_{j}=\left.\psi_{j}\right|^{i} K_{j}$,

(2) if $v$ is a vertex of $K_{i}, \psi_{i}(v) \in f^{-1}(\phi(v))$,

(3) if $\sigma$ is a $j$-simplex of $K_{i}^{i}$ and $k=\operatorname{dim} \operatorname{st}\left(\sigma, K_{i}^{i}\right)$, then $\phi\left(\operatorname{st}\left(\sigma, K_{i}\right)\right) \cup f \psi_{i}(\sigma) \subset U$, for some $U \in \alpha_{k-j}$.

[Note that $\psi_{0}: K_{0}^{0} \rightarrow X$ satisfies these conditions since if $\sigma$ is a 0 -simplex of $K_{0}^{0}$ the dimension of $\operatorname{st}\left(\sigma, K_{0}^{0}\right)$ is 0 and the fact that $K_{0}$ is a $\left(0, \alpha_{0}\right)$-subdivision of $K$ implies that $\phi\left(\operatorname{st}\left(\sigma, K_{0}\right)\right) \cup f \psi_{0}(\sigma) \subset U$ for some $U \in \alpha_{0}$.]

We wish now to define $\psi_{n+1}: K_{n+1}^{n+1} \rightarrow X$ satisfying conditions (1) - (3) for $i=n+1$. For each vertex $v$ of $K_{n+1}$, let 


$$
\psi_{n+1}(v)=\left\{\begin{array}{l}
\psi_{n}(v), \text { if } v \text { is a vertex of }{ }^{n} K_{n} \\
\psi^{\prime}(v), \text { if } v \in J
\end{array}\right.
$$

an arbitrary element of $f^{-1}(\phi(v))$, otherwise

Assume (subinductive statement) that $\psi_{n+1} \mid K_{n+1}^{r}$ has been defined so that

$$
\begin{aligned}
& \text { (1') } \psi_{n+1}\left|J \cap K_{n+1}^{r}=\psi^{\prime}\right| J \cap K_{n+1}^{r} \quad \text { and } \quad \psi_{n+1} \mid{ }^{n} K_{n} \cap K_{n+1}^{r}= \\
& \psi_{n} \mid{ }^{n} K_{n} \cap K_{n+1}^{r}, \\
& \text { (2') if } v \text { is a vertex of } K_{n+1}, \psi_{n+1}(v) \in f^{-1}(\phi(v)), \\
& \text { (3') if } \sigma \text { is a } j \text {-simplex of } K_{n+1}^{r} \text { and } k=\operatorname{dim} \operatorname{st}\left(\sigma, K_{n+1}^{n+1}\right) \text {, then } \\
& \phi\left(\operatorname{st}\left(\sigma, K_{n+1}\right)\right) \cup f \psi_{n+1}(\sigma) \subset U \text {, for some } U \in \alpha_{k-j} .
\end{aligned}
$$

[Note that $\psi_{n+1} \mid K_{n+1}^{0}$ has been defined in such a manner that properties $\left(1^{\prime}\right)-\left(3^{\prime}\right)$ are satisfied. Properties $\left(1^{\prime}\right)$ and $\left(2^{\prime}\right)$ follow immediately from the definition. Let $v$ be a simplex of $K_{n+1}^{0}$. If $v$ is a vertex of ${ }^{n} K_{n}$, then property ( $\left.3^{\prime}\right)$ follows from the fact that $\psi_{n}$ satisfies property (3) of the main inductive statement since in this case $\operatorname{dim} \operatorname{st}\left(v, K_{n+1}^{n+1}\right)=$ $\operatorname{dimst}\left(v, K_{n}^{n}\right)$. Suppose $v$ is not a vertex of ${ }^{n} K_{n}$. By the remark following Lemma $1, \phi\left(\operatorname{st}\left(v, K_{n+1}\right)\right)$ is contained in some element of $\alpha_{n+1}$ and hence property $\left(3^{\prime}\right)$ is again satisfied.]

Now let $\sigma$ be an $(r+1)$-simplex of $K_{n+1}$. If $\sigma$ is a subset of $J$, let $\psi_{n+1}\left|\sigma=\psi^{\prime}\right| \sigma$. If $\sigma<{ }^{n} K_{n}$, let $\psi_{n+1}\left|\sigma=\psi_{n}\right| \sigma$. Otherwise, let $k=$ $\operatorname{dim} \operatorname{st}\left(\sigma, K_{n+1}^{n+1}\right)$. For each $r$-simplex, $\tau$, in $\partial \sigma$, there is a $u_{\tau} \in \alpha_{k-r}$ containing $\phi\left(\operatorname{st}\left(\tau, K_{n+1}\right)\right) \cup f \psi_{n+1}(\tau)$. Let $\tau^{\prime}$ be a fixed $r$-simplex in $\partial \sigma$ and note that $\psi_{n+1}(\partial \sigma) \subset f^{-1}\left(\operatorname{st}\left(u_{\tau^{\prime}}, \alpha_{k-r}\right)\right)$. Since $\alpha_{k-r}$ is a $U V^{\infty}$ star refinement of $\alpha_{k-r-1}$, there is a $U \in \alpha_{k-r-1}=\alpha_{k-(r+1)}$ containing $\operatorname{st}\left(U_{\tau^{\prime}}, \alpha_{k-r}\right)$ and an extension of $\psi_{n+1} \mid \partial \sigma$ which maps $\sigma$ into $f^{-1}(U)$. We call this extension $\psi_{n+1}$ and note that $\phi\left(\operatorname{st}\left(\sigma, K_{n+1}\right)\right) \cup f \psi_{n+1}(\sigma) \subset U$. In this manner, extend $\psi_{n+1}$ to $K_{n+1}^{r+1}$ and note that conditions $\left(1^{\prime}\right)-\left(3^{\prime}\right)$ are satisfied. This completes the subinductive argument and hence the main inductive argument.

We now define $\psi: K \rightarrow X$ by $\psi(x)=\lim _{n \rightarrow \infty} \psi_{n}(x)$. For any $x \in K$, the local finiteness of $K$ assures that there exists an integer $N$ so that $x \in{ }^{N} K_{N}$. Hence for $n \geqq N, \psi_{n}(x)=\psi_{N}(x)$. Therefore $\psi$ is welldefined and continuous. Let $x \in K$ and let $\sigma$ be a simplex of maximal dimension containing $x$. Then there exists an integer $N$ such that $|\sigma| \subset^{N} K_{N}$. Choose a simplex $B$ in ${ }^{N} K_{N}$ containing $x$ and note that $\psi(x)=\psi_{N}(x)$. By inductive statement (3), there is an open set $U \in \alpha_{i}$, for some $i \geqq 0$, such that $\phi\left(\operatorname{st}\left(B, K_{N}\right)\right) \cup f \psi(B) \subset U$. Since $\alpha_{i}$ refines $\alpha_{0}=\alpha$, there is a $V \in \alpha$ such that $\{\phi(x)\} \cup\{f \psi(x)\} \subset V$. Since $\psi$ extends $\psi^{\prime}$, this completes the proof of Lemma 3 . 
REMARK. By a slightly more cumbersome process, $\psi$ can be chosen so that $f \psi$ is a $\alpha$-homotopic to $\phi$.

TheOREM. Let $X$ and $Y$ be separable ANRs and $f: X \rightarrow Y$ be a closed $U V^{\infty}$-map. Then $f$ is a fine homotopy equivalence.

Proof. Let $\alpha$ be an open cover of $Y$, Let $\alpha_{1}$ be a star ${ }^{5}$ refinement of $\alpha$ and $\alpha_{2}$ a star refinement of $\alpha_{1}$. Let $\beta$ be an open refinement of $\alpha_{2}$ such that any two $\beta$-near maps from any space into $Y$ are $\alpha_{2}$-homotopic (such refinements exists since $Y$ is an ANR, c.f. [4]).

By Hanner's characterization of separable ANRs (c.f. [4]), there exist a locally finite polyhedron $Q$ and maps $c: Q \rightarrow Y$ and $s: Q \rightarrow Y$ with property that $s c_{2}^{\alpha_{2}} i d_{Y}$. By Lemma 3 , there is a map $v: Q \rightarrow X$ such that $f v$ is $\beta$-near $s$. Define $g: Y \rightarrow X$ by $g=v c$. Note that $f g$ is $\beta$-near $s c$ and hence $f g \stackrel{\alpha_{2}}{=} s c$. But $s c \stackrel{\alpha_{2}}{\cong} i d_{Y}$ and hence $f g \stackrel{\alpha_{2}}{\simeq} i d_{Y}$. Denote this $\alpha_{1}$-homotopy by $h$; then, $h: Y \times I \rightarrow Y$ is a $\alpha_{1}$-homotopy with $h_{0}=i d_{Y}$ and $h_{1}=f g$.

It remains to be shown that $g f$ is $f^{-1}(\alpha)$ homotopic to $i d_{x}$.

Choose a locally finite polyhedron, $P$, maps $b: \rightarrow P$ and $r: P \rightarrow X$ and a homotopy $W: X \times I \rightarrow X$ with the following properties:

(a) $W_{0}=r b$ and $W_{1}=i d_{X}$

(b) $W$ is limited by $f^{-1}\left(\alpha_{1}\right)$ and by $(g f)^{-1}\left(f^{-1}\left(\alpha_{1}\right)\right)$.

Next, define $H: P \times I \rightarrow Y$ by $H(p, t)=h_{t}(f r(p))$ and note that $H(p, 0)=f r(p)$ and $H(p, 1)=f g f r(p)$. Define $G^{\prime}: P \times\{0,1\} \rightarrow X$ by $G^{\prime}(p, 0)=r(p), G^{\prime}(p, 1)=g f r(p)$. Then by Lemma 3 there is a map $G: P \times I \rightarrow X$ extending $G^{\prime}$ with the property that $f G$ is $\alpha_{1}$-near $H$.

Define $\psi: X \times I \rightarrow X$ by $\psi(x, t)=G(b(x), t)$.

Note that: $\psi_{0}(x)=G(b(x), 0)=G^{\prime}(b(x), 0)=r b(x)$ and $\psi_{1}(x)=$ $G(b(x), 1)=G^{\prime}(b(x), 1)=g f r b(x)$.

Now, $W$ is a homotopy taking $r b$ to $i d_{x}$ and is limited by $f^{-1}\left(\alpha_{1}\right)$. Also, since $W$ is limited by $(g f)^{-1}\left(f^{-1}\left(\alpha_{1}\right)\right), g f W: X \times I \rightarrow X$, defined by $g f W(x, t)=g f(W(x, t))$, is a homotopy taking $g f r b$ to $g f$ and is limited by $f^{-1}\left(\alpha_{1}\right)$.

Recall that $\alpha_{1}$ is a $\operatorname{star}^{5}$ refinement of $\alpha$. Therefore, to show that $i d_{X} \stackrel{f^{-1}(\alpha)}{=} g f$, it suffices to show that $f \psi: X \times I \rightarrow Y$ is limited by star $^{3}$ $\left(\alpha_{1}\right)$. Fix $x \in X$. Since the homotopy $h$ is limited by $\alpha_{1}$, there exists $U \in \alpha_{1}$ with $h(f(x) \times I) \subset U$. we claim that $f(\psi(x \times I)) \subset \operatorname{st}^{3}(U)$.

Fix $t \in I$. Recall $f(\psi(x, t))=f(G(b(x), t))$. Thus there exists $U^{\prime} \in \alpha_{1}$ such that $f^{-1}\left(U^{\prime}\right)$ contains $x$ and $r b(x)$. Hence $f(x)$ and $f r b(x)$ are elements of $U^{\prime}$ and $U \cap U^{\prime} \neq \phi$. Since $h$ is limited by $\alpha_{1}$, we can choose $U^{\prime \prime} \in \alpha_{1}$ so that $h_{t} f r b(x)$ and $f r b(x)$ are elements of $U^{\prime \prime}$. Note that $U^{\prime \prime} \cap U^{\prime} \neq \phi$. Also, there exists $U^{\prime \prime \prime} \in \alpha_{1}$ containing $H(b(x), t)$ and $f(G(b(x), t))$, since $f G$ is $\alpha_{1}$-near $H$. But $H(b(x), t)=$ 
$h_{t} f r b(x)$. Hence $U^{\prime \prime \prime} \cap U^{\prime \prime} \neq \phi$ and we have completed the proof of the theorem by showing that $f \psi: X \times I \rightarrow Y$ is limited by $\operatorname{star}^{3}\left(\alpha_{1}\right)$.

Added in proof. I would like to thank Bob Edwards for some suggestions concerning this paper and for pointing out that George Kozlowski [Images of ANR's, to appear] has shown that a $U V^{\infty}$-map between ANR's is a homotopy equivalence.

REMARK. If in addition it is assumed that $X$ and $Y$ are locally compact and $f$ is a proper map it follows immediately that $f$ is a proper fine homotopy equivalence.

\section{REFERENCES}

1. R. D. Anderson and N. Kroonenberg, Open problems in Infinite-dimensional topology, to appear.

2. S. Armentrout and T. Price, Decompositions into compact sets with UV properties, Trans. Amer. Math. Soc., 141 (1969), 433-442.

3. J. Dugundji, Topology, Allyn and Bacon, Boston, 1966.

4. S. T. Hu, Theory of Retracts, Wayne State University Press, Detroit, 1965.

5. G. Kozlowski, Factorization of certain maps up to homotopy, Proc. Amer. Math. Soc., 21 (1969), 88-92.

6. R. C. Lacher, Cell-like mappings I, Pacific J. Math., 30 (1969).

7. R. C. Lacher, Cell-like mappings of ANRs, Bull. Amer. Math. Soc., 74 (1968), 933-935.

8. S. Smale, A Vietoris mapping theorem for homotopy, Proc. Amer. Math. Soc., 8 (1957), 604-610.

9. J. West, Compact ANR's have finite type, Bull. Amer. Math. Soc., 81 (1975), 163-165.

Received March 12, 1974 and in revised form July 20, 1974.

UNIVERSITY OF TENNESSEE 



\section{PACIFIC JOURNAL OF MATHEMATICS}

\section{EDITORS}

RICHARD ARENS (Managing Editor)

University of California

Los Angeles, California 90024

\section{J. DugundJI}

Department of Mathematics University of Southern California Los Angeles, California 90007

D. Gilbarg and J. Milgram

Stanford University

Stanford, California 94305

\section{ASSOCIATE EDITORS}
E. F. BECKENBACH
B. H. NeumanN
F. WoLF
K. YoshiDA

\section{SUPPORTING INSTITUTIONS}

\author{
UNIVERSITY OF BRITISH COLUMBIA \\ CALIFORNIA INSTITUTE OF TECHNOLOGY \\ UNIVERSITY OF CALIFORNIA \\ MONTANA STATE UNIVERSITY \\ UNIVERSITY OF NEVADA \\ NEW MEXICO STATE UNIVERSITY \\ OREGON STATE UNIVERSITY \\ UNIVERSITY OF OREGON \\ OSAKA UNIVERSITY
}

\author{
UNIVERSITY OF SOUTHERN CALIFORNIA \\ STANFORD UNIVERSITY \\ UNIVERSITY OF TOKYO \\ UNIVERSITY OF UTAH \\ WASHINGTON STATE UNIVERSITY \\ UNIVERSITY OF WASHINGTON \\ AMERICAN MATHEMATICAL SOCIETY
}

The Supporting Institutions listed above contribute to the cost of publication of this Journal, but they are not owners or publishers and have no responsibility for its contents or policies.

Mathematical papers intended for publication in the Pacific Journal of Mathematics should be in typed form or offset-reproduced (not dittoed), double spaced with large margins. Underline Greek letters in red, German in green, and script in blue. The first paragraph or two must be capable of being used separately as a synopsis of the entire paper. Items of the bibliography should not be cited there unless absolutely necessary, in which case they must be identified by author and Journal, rather than by item number. Manuscripts, in duplicate, may be sent to any one of the four editors. Please classify according to the scheme of Math. Reviews, Index to Vol. 39. All other communications should be addressed to the managing editor, or Elaine Barth, University of California, Los Angeles, California, 90024.

100 reprints are provided free for each article, only if page charges have been substantially paid. Additional copies may be obtained at cost in multiples of 50 .

The Pacific Journal of Mathematics is issued monthly as of January 1966. Regular subscription rate: $\$ 72.00$ a year (6 Vols., 12 issues). Special rate: $\$ 36.00$ a year to individual members of supporting institutions.

Subscriptions, orders for back numbers, and changes of address should be sent to Pacific Journal of Mathematics, 103 Highland Boulevard, Berkeley, California, 94708.

PUBLISHED BY PACIFIC JOURNAL OF MATHEMATICS, A NON-PROFIT CORPORATION Printed at Jerusalem Academic Press, POB 2390, Jerusalem, Israel.

$$
\begin{gathered}
\text { Copyright (C) } 1975 \text { Pacific Journal of Mathematics } \\
\text { All Rights Reserved }
\end{gathered}
$$




\section{Pacific Journal of Mathematics}

\section{Vol. 58, No. $2 \quad$ April, 1975}

Zvi Artstein and John Allen Burns, Integration of compact set-valued functions . . . . . . . . . 297

Mark Benard, Characters and Schur indices of the unitary reflection group $[321]^{3} \ldots \ldots \ldots . .309$

Simeon M. Berman, A new characterization of characteristic functions of absolutely continuous

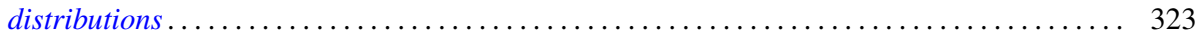

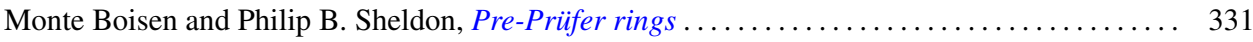

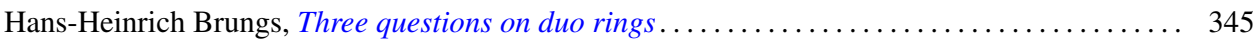

Iracema M. Bund, Birnbaum-Orlicz spaces of functions on groups................. 351

John D. Elwin and Donald R. Short, Branched immersions between 2-manifolds of higher

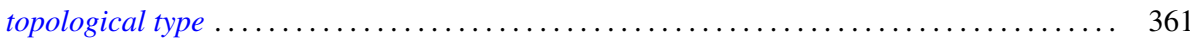

Eric Friedlander, Extension functions for rank 2, torsion free abelian groups . .......... 371

Jon Froemke and Robert Willis Quackenbush, The spectrum of an equational class of

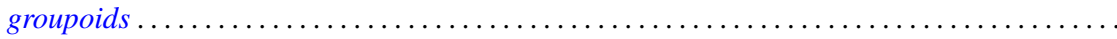

Barry J. Gardner, Radicals of supplementary semilattice sums of associative rings ...........

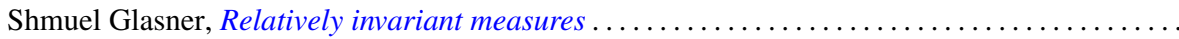

George Rudolph Gordh, Jr. and Sibe Mardesic, Characterizing local connectedness in inverse

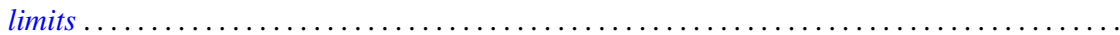

Siegfried Graf, On the existence of strong liftings in second countable topological spaces......

Stanley P. Gudder and D. Strawther, Orthogonally additive and orthogonally increasing

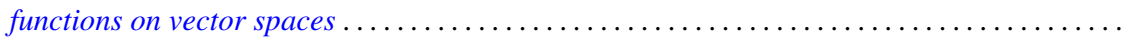

Darald Joe Hartfiel and Carlton James Maxson, A characterization of the maximal monoids and

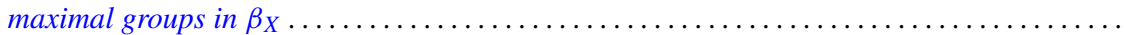

Robert E. Hartwig and S. Brent Morris, The universal flip matrix and the generalized faro-shuffle. .

William Emery Haver, Mappings between ANRs that are fine homotopy equivalences. .

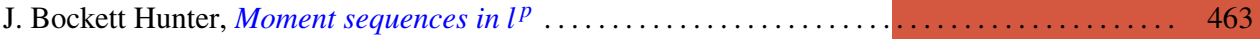

Barbara Jeffcott and William Thomas Spears, Semimodularity in the completion of a poset.... 467

Jerry Alan Johnson, A note on Banach spaces of Lipschitz functions . . . . . . . . . . . . 475

David W. Jonah and Bertram Manuel Schreiber, Transitive affine transformations on

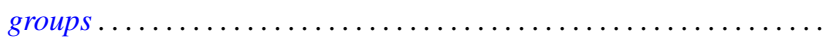

Karsten Juul, Some three-point subset properties connected with Menger's characterization of

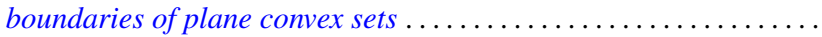

Ronald Brian Kirk, The Haar integral via non-standard analysis . . . . . . . . . . . . . 517

Justin Thomas Lloyd and William Smiley, On the group of permutations with countable

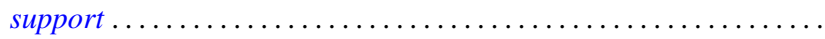

Erwin Lutwak, Dual mixed volumes .................................. 531

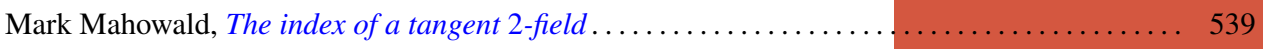

Keith Miller, Logarithmic convexity results for holomorphic semigroups . . . . . . . . . . . . 549

Paul Milnes, Extension of continuous functions on topological semigroups . . . . . . . . . . 553

Kenneth Clayton Pietz, Cauchy transforms and characteristic functions ................ 563

James Ted Rogers Jr., Whitney continua in the hyperspace $C(X) \ldots \ldots \ldots \ldots \ldots \ldots \ldots \ldots .569$

Jean-Marie G. Rolin, The inverse of a continuous additive functional . . . . . . . . . . . . 585

William Henry Ruckle, Absolutely divergent series and isomorphism of subspaces . ........ 605

Rolf Schneider, A measure of convexity for compact sets . ..................... 617

Alan Henry Schoenfeld, Continous measure-preserving maps onto Peano spaces .......... 627

V. Merriline Smith, Strongly superficial elements .......................... 643

Roger P. Ware, A note on quadratic forms over Pythagorean fields . . . . . . . . . . . . . . 651

Roger Allen Wiegand and Sylvia Wiegand, Finitely generated modules over Bezout rings . . . . 655

Martin Ziegler, A counterexample in the theory of definable automorphisms . . . . . . . . . 665 